\title{
SMOOTHED PROJECTIONS IN FINITE ELEMENT EXTERIOR CALCULUS
}

\author{
SNORRE H. CHRISTIANSEN AND RAGNAR WINTHER
}

\begin{abstract}
The development of smoothed projections, constructed by combining the canonical interpolation operators defined from the degrees of freedom with a smoothing operator, has proved to be an effective tool in finite element exterior calculus. The advantage of these operators is that they are $L^{2}$ bounded projections, and still they commute with the exterior derivative. In the present paper we generalize the construction of these smoothed projections, such that also non-quasi-uniform meshes and essential boundary conditions are covered. The new tool introduced here is a space-dependent smoothing operator that commutes with the exterior derivative.
\end{abstract}

\section{INTRODUCTION}

Differential forms and exterior calculus represent an area of growing importance for the understanding of the finite element method. This was first recognized by Bossavit [5], where the equivalence between the Nédélec $H$ (curl) elements [11, 12 ] and the Whitney forms [15] is pointed out. The relation between exterior calculus and finite element discretizations, in particular for electromagnetic problems, is also discussed by Hiptmair [8, 9, 10, and further developed in [1, 2, 3, 4]. The theory presented here is closely related to the presentation given in [4].

By combining the canonical interpolation operators onto the standard finite element spaces of exterior calculus with a suitable smoothing operator one can obtain modified operators with desirable properties. More precisely, these modified interpolation operators are projections, they commute with the exterior derivative, and they are uniformly $L^{2}$ bounded. This is in contrast to the canonical interpolation operators, defined directly from the degrees of freedom, which are only defined for functions with higher-order regularity. As a consequence of the construction of the new projections the proper mixed finite element discretizations of the Hodge Laplacian problem are easily seen to be stable without appealing to extra regularity. This means that a technical problem, frequently encountered in earlier studies of mixed finite element methods, is avoided. Furthermore, the new projections are essential

Received by the editor December 20, 2006 and, in revised form, March 22, 2007.

2000 Mathematics Subject Classification. Primary 65N30, 53A45.

Key words and phrases. Exterior calculus, finite elements, interpolation operators.

This research was supported by the Norwegian Research Council. The first author acknowledges that this work, conducted as part of the award "Numerical analysis and simulations of geometric wave equations" made under the European Heads of Research Councils and European Science Foundation EURYI (European Young Investigator) Awards scheme, was supported by funds from the Participating Organizations of EURYI and the EC Sixth Framework Program. 
for establishing that the discretization preserves the dimensions of the cohomology groups.

The first suggestion to use a combination of a smoothing operator and the canonical interpolation operator, to overcome the lack of boundedness of the latter, seems to be in Dodziuk and Patodi [7, where a heat kernel is used as a smoothing operator. A treatment of such smoothed projections more in line with the techniques of numerical analysis, and highly influenced by earlier contributions of Christiansen [6] and Schöberl [14, is presented in [4, Section 5]. However, the analysis given in [4] of these smoothed projections is not developed in full generality. First of all, the analysis given there requires so-called quasi-uniform meshes, a condition well known to be undesired in many practical computations. Secondly, the theory given in [4] only treats the spaces of differential forms that are without restrictions of the traces on the boundary of the domain. In other words, the theory only treats boundary value problems with natural boundary conditions, while problems with essential boundary conditions are not covered. The purpose of the present paper is to generalize the construction done in [4] so that we allow non-quasi-uniform meshes, and such that essential boundary conditions are covered. Here we should mention that non-quasi-uniform meshes and essential boundary conditions are also covered by the smoothed projections introduced in 14 .

In Section 2 we recall some basic properties of exterior calculus, while the construction of the spaces of discrete differential forms is outlined in Section 3 . The new tool introduced here, compared to the theory developed in 4, is a spacedependent smoothing operator that commutes with the exterior derivative. This operator is introduced and discussed in Section 4. The construction of the smoothed projections is then completed in Section [5] and the main theoretical results are also derived here. Corresponding results for the case of essential boundary conditions are then presented in Section 6 .

\section{NotATION AND PRELIMINARIES}

The notation used in this paper corresponds closely to the notation used in [4, and we refer to this paper and the references given there for more details on exterior calculus. In particular, if $T \subset \mathbb{R}^{n}$, then $\mathcal{P}_{r}(T)$ denotes the set of scalar polynomials of degree less than or equal to $r$ defined on $T$, and we will use $B_{\delta}(x) \subset \mathbb{R}^{n}$ to denote the ball with centre at $x$ and radius $\delta$. For any simplex $T \in \mathbb{R}^{n}$ we let $\Delta_{j}(T)$ denote the set of subsimplexes of dimension $j$, while $\Delta(T)$ is the set of all subsimplexes. If $X$ and $Y$ are normed linear spaces, then $\mathrm{t}(X, Y)$ is the space of bounded linear operators from $X$ to $Y$, and $\|\cdot\|_{\mathrm{E}(X, Y)}$ denotes the corresponding norm. Throughout the paper we will assume that $\Omega$ is a fixed bounded polyhedral domain in $\mathbb{R}^{n}$ with boundary $\partial \Omega$. The symbol Alt ${ }^{k}$ is used to denote the set of alternating $k$-forms on $\mathbb{R}^{n}$. Hence, $\mathrm{Alt}^{k}$ is a linear space of dimension $\left(\begin{array}{l}n \\ k\end{array}\right)$. The exterior product, or the wedge product, maps $\mathrm{Alt}^{j} \times \mathrm{Alt}^{k}$ into $\mathrm{Alt}^{j+k}$. For $\omega \in \mathrm{Alt}^{j}$, $\eta \in \mathrm{Alt}^{k}$, and given vectors $v_{1}, v_{2}, \ldots, v_{j+k} \in \mathbb{R}^{n}$ the exterior product $\omega \wedge \eta \in \mathrm{Alt}^{j+k}$ is given by

$$
(\omega \wedge \eta)\left(v_{1}, \ldots, v_{j+k}\right)=\sum_{\sigma}(\operatorname{sign} \sigma) \omega\left(v_{\sigma(1)}, \ldots, v_{\sigma(j)}\right) \eta\left(v_{\sigma(j+1)}, \ldots, v_{\sigma(j+k)}\right),
$$

where the sum is over all permutations $\sigma$ of $\{1, \ldots, j+k\}$, for which $\sigma(1)<\sigma(2)<$ $\cdots<\sigma(j)$ and $\sigma(j+1)<\sigma(j+2)<\cdots<\sigma(j+k)$. If $d x_{i} \in \mathrm{Alt}^{1}$ is given by $d x_{i}\left(e_{j}\right)=\delta_{i j}$, where $\left\{e_{1}, e_{2}, \ldots, e_{n}\right\}$ is the standard basis for $\mathbb{R}^{n}$, then a basis for 
$\mathrm{Alt}^{k}$ is given by

$$
\left\{d x_{\sigma(1)} \wedge d x_{\sigma(2)} \wedge \cdots \wedge d x_{\sigma(k)} \mid \sigma \in \Sigma(k, n)\right\},
$$

where $\Sigma(k, n)$ is the set of increasing maps $\{1,2, \ldots, k\} \rightarrow\{1,2, \ldots, n\}$.

We will use $\Lambda^{k}(\Omega)$ to denote the space of smooth $k$-forms on $\Omega$, i.e. $\Lambda^{k}(\Omega):=$ $C^{\infty}\left(\Omega ; \mathrm{Alt}^{k}\right)$. Hence, any element $\omega \in \Lambda^{k}(\Omega)$ has a unique representation of the form

$$
\omega_{x}=\sum_{\sigma \in \Sigma(k, n)} a_{\sigma}(x) d x_{\sigma(1)} \wedge d x_{\sigma(2)} \wedge \cdots \wedge d x_{\sigma(k)},
$$

where $a$ is a scalar smooth function, i.e. $a \in C^{\infty}(\Omega)$. The corresponding space $C\left(\Omega ;\right.$ Alt $\left.^{k}\right)$ will be denoted $C \Lambda^{k}(\Omega)$. The exterior derivative $d: \Lambda^{k}(\Omega) \rightarrow \Lambda^{k+1}(\Omega)$ is given by

$$
\begin{aligned}
d\left(\sum_{\sigma} a_{\sigma} d x_{\sigma(1)} \wedge d x_{\sigma(2)} \wedge \cdots\right. & \left.\wedge d x_{\sigma(k)}\right) \\
& =\sum_{\sigma} \sum_{i=1}^{n} \frac{\partial a_{\sigma}}{\partial x_{i}} d x_{i} \wedge d x_{\sigma(1)} \wedge d x_{\sigma(2)} \wedge \cdots \wedge d x_{\sigma(k)} .
\end{aligned}
$$

The exterior derivative has the property that $d \circ d=0$, and the classical de Rham complex is given by the maps and the spaces

$$
\Lambda^{0}(\Omega) \stackrel{d}{\rightarrow} \Lambda^{1}(\Omega) \stackrel{d}{\rightarrow} \cdots \stackrel{d}{\rightarrow} \Lambda^{n}(\Omega) .
$$

Furthermore, the exterior derivative satisfies the Leibniz rule

$$
d(\omega \wedge \eta)=d \omega \wedge \eta+(-1)^{j} \omega \wedge d \eta, \quad \omega \in \Lambda^{j}(\Omega), \eta \in \Lambda^{k}(\Omega) .
$$

A key tool, which we will use repeatedly in the analysis below, is the pullback of differential forms. If $\phi$ is a smooth map from $\Omega \subset \mathbb{R}^{n}$ to $\Omega^{\prime} \subset \mathbb{R}^{m}$, then the pullback $\phi^{*}: \Lambda^{k}\left(\Omega^{\prime}\right) \rightarrow \Lambda^{k}(\Omega)$ is given by

$$
\left(\phi^{*} \omega\right)_{x}\left(v_{1}, v_{2}, \ldots, v_{k}\right)=\omega_{\phi(x)}\left(D \phi_{x}\left(v_{1}\right), D \phi_{x}\left(v_{2}\right), \ldots, D \phi_{x}\left(v_{k}\right)\right),
$$

where the linear map $D \phi_{x}: \mathbb{R}^{n} \rightarrow \mathbb{R}^{m}$ is the derivative of $\phi$ at $x$. The pullbacks commute with the exterior derivative, i.e.

$$
\phi^{*}(d \omega)=d\left(\phi^{*} \omega\right), \quad \omega \in \Lambda^{k}\left(\Omega^{\prime}\right) .
$$

Furthermore,

We also recall that if

$$
\phi^{*}(\omega \wedge \eta)=\phi^{*} \omega \wedge \phi^{*} \eta
$$

$$
\omega=a d x_{1} \wedge d x_{2} \wedge \cdots \wedge d x_{n} \in \Lambda^{n}(\Omega),
$$

then $\int_{\Omega} \omega$ is defined to be the ordinary multiple integral of the function $a$ over $\Omega$. As a consequence, if $\phi: \Omega \rightarrow \Omega^{\prime} \subset \mathbb{R}^{n}$ is an orientation-preserving diffeomorphism, then

$$
\int_{\Omega} \phi^{*} \omega=\int_{\Omega^{\prime}} \omega, \quad \omega \in \Lambda^{n}\left(\Omega^{\prime}\right)
$$

If $\omega \in \Lambda^{n-1}(\Omega)$, then the formula (2.3) can also be used to define the integral of the trace of $\omega, \operatorname{Tr} \omega$, over the boundary $\partial \Omega$. Here $\operatorname{Tr} \omega$ corresponds to an alternating $(n-1)$-form on $\partial \Omega$ acting on tangent vectors. Stokes' theorem now takes the form

$$
\int_{\Omega} d \omega=\int_{\partial \Omega} \operatorname{Tr} \omega, \quad \omega \in \Lambda^{n-1}(\Omega) .
$$


Combining Stokes' theorem and the Leibniz rule (2.2) we also obtain the integration by parts identity

$$
\int_{\Omega} d \omega \wedge \eta=(-1)^{k-1} \int_{\Omega} \omega \wedge d \eta+\int_{\partial \Omega} \operatorname{Tr} \omega \wedge \operatorname{Tr} \eta,
$$

for $\omega \in \Lambda^{k}(\Omega)$ and $\eta \in \Lambda^{n-k-1}(\Omega)$.

The Hilbert space $L^{2} \Lambda^{k}(\Omega) \supset \Lambda^{k}(\Omega)$ can be identified as all $\omega$ of the form (2.1), where the functions $a_{\sigma}$ are elements of $L^{2}(\Omega)$. The corresponding inner product is given by

$$
\langle\omega, \eta\rangle_{L^{2} \Lambda^{k}}=\sum_{\sigma \in \Sigma(k, n)} a_{\sigma} b_{\sigma},
$$

where $\eta=\sum_{\sigma} b_{\sigma} d x_{\sigma(1)} \wedge \cdots \wedge d x_{\sigma(k)}$. Similarly, the Sobolev space $H^{s} \Lambda^{k}(\Omega)$ consists of all $\omega$ of the form (2.1), where $a_{\sigma} \in H^{s}(\Omega)$, while the space $H \Lambda^{k}(\Omega)$ is defined by

$$
H \Lambda^{k}(\Omega)=\left\{\omega \in L^{2} \Lambda^{k}(\Omega) \mid d \omega \in L^{2} \Lambda^{k+1}(\Omega)\right\} .
$$

Note that we have $H \Lambda^{0}(\Omega)=H^{1} \Lambda^{0}(\Omega), H \Lambda^{n}(\Omega)=L^{2} \Lambda^{n}(\Omega)$, and in general $H^{1} \Lambda^{k}(\Omega) \subset H \Lambda^{k}(\Omega) \subset L^{2} \Lambda^{k}(\Omega)$. The $L^{2}$ de Rham complex, or the Sobolev version of the de Rham complex, is the sequence of maps and spaces given by

$$
H \Lambda^{0}(\Omega) \stackrel{d}{\rightarrow} H \Lambda^{1}(\Omega) \stackrel{d}{\rightarrow} \cdots \stackrel{d}{\rightarrow} H \Lambda^{n}(\Omega) .
$$

We recall from [4, Section 2] that the map $\omega \mapsto \operatorname{Tr} \omega$ is continuous as a map from $H \Lambda^{k}(\Omega)$ to $H^{-1 / 2} \Lambda^{k}(\partial \Omega)$. As a consequence, the space $\stackrel{\circ}{H} \Lambda^{k}(\Omega) \subset H \Lambda^{k}(\Omega)$ given by

$$
\stackrel{\circ}{H} \Lambda^{k}(\Omega)=\left\{\omega \in H \Lambda^{k}(\Omega) \mid \operatorname{Tr} \omega=0\right\}
$$

is well defined. The corresponding de Rham complex, involving these spaces with essential boundary conditions, takes the form

$$
\stackrel{\circ}{H} \Lambda^{0}(\Omega) \stackrel{d}{\rightarrow} \stackrel{\circ}{H} \Lambda^{1}(\Omega) \stackrel{d}{\rightarrow} \cdots \stackrel{d}{\rightarrow} \stackrel{\circ}{H} \Lambda^{n}(\Omega) .
$$

\section{Discrete Differential Forms}

In finite element exterior calculus we are constructing proper discrete subcomplexes of the complexes (2.6) and (2.7). In order to define these finite element spaces we will assume that the polyhedral domain $\Omega$ is partitioned into a finite set of $n$-simplices $\mathcal{T}$. These $n$-simplices determine a simplicial decomposition of $\Omega$, i.e. their union is the closure of $\Omega$, and the intersection of any two is either empty or a common subsimplex of each. Adopting the terminology of the two-dimensional case, we will refer to $\mathcal{T}$ as a triangulation of $\Omega$.

We consider a family of triangulations $\left\{\mathcal{T}_{h}\right\}$ of $\Omega$ indexed by the discretization parameter

$$
h=\max _{T \in \mathcal{T}_{h}} h_{T}, \quad \text { where } h_{T}=\operatorname{diam} T .
$$

We assume that the discretization parameter runs over a set of positive values bounded from above and accumulating at zero. Furthermore, the family $\left\{\mathcal{T}_{h}\right\}$ is assumed to be shape regular; i.e., there exists a mesh regularity constant $C_{\text {mesh }}>0$, independent of the triangulation $\mathcal{T}_{h}$, such that

$$
h_{T}^{n} \leq C_{\text {mesh }}|T|, \quad T \in \mathcal{T}_{h},
$$

where $|T|$ denotes the volume of the simplex $T$. However, no quasi-uniformity condition is assumed; i.e., there is no uniform bound on $h / h_{T}$, for $T \in \mathcal{T}_{h}$. We 
observe that a simple consequence of (3.1) is the existence of another uniform regularity constant $C_{\text {mesh }}^{\prime}>0$ such that

$$
h_{T} \leq C_{\text {mesh }}^{\prime}|e|, \quad e \in \Delta_{1}(T), T \in \mathcal{T}_{h},
$$

where $|e|$ is the length of the edge $e$.

The discrete spaces which we will consider will consist of piecewise polynomial differential forms; i.e., the elements reduce to polynomial differential forms on each simplex $T$. The space $\mathcal{P}_{r} \Lambda^{k}(T)$ is simply given as all functions of the form (2.1), where the coefficients $a_{\sigma} \in \mathcal{P}_{r}(T)$. Hence, we obtain

$$
\operatorname{dim} \mathcal{P}_{r} \Lambda^{k}(T)=\left(\begin{array}{c}
n+r \\
n
\end{array}\right)\left(\begin{array}{l}
n \\
k
\end{array}\right)=\left(\begin{array}{c}
r+k \\
k
\end{array}\right)\left(\begin{array}{l}
n+r \\
n-k
\end{array}\right) .
$$

As an alternative to these complete polynomial spaces of degree $r$, we can also consider the spaces $\mathcal{P}_{r}^{-} \Lambda^{k}(T)$, for $r \geq 1$. These spaces are given as

$$
\mathcal{P}_{r}^{-} \Lambda^{k}(T)=\mathcal{P}_{r-1} \Lambda^{k}(T)+\kappa \mathcal{P}_{r-1} \Lambda^{k+1}(T) .
$$

Here $\kappa: \mathcal{P}_{r-1} \Lambda^{k+1}(T) \rightarrow \mathcal{P}_{r} \Lambda^{k}(T)$ is the Kozul operator defined by

$$
(\kappa \omega)_{x}\left(v_{1}, v_{2}, \ldots, v_{k}\right)=\omega_{x}\left(x, v_{1}, \ldots, v_{k}\right)
$$

for $v_{1}, v_{2}, \ldots, v_{k} \in \mathbb{R}^{n}$. It can be seen that $\mathcal{P}_{r}^{-} \Lambda^{0}(T)=\mathcal{P}_{r} \Lambda^{0}(T), \mathcal{P}_{r}^{-} \Lambda^{n}(T)=$ $\mathcal{P}_{r-1} \Lambda^{n}(T)$, and in general $\mathcal{P}_{r-1} \Lambda^{k}(T) \subset \mathcal{P}_{r}^{-} \Lambda^{k}(T) \subset \mathcal{P}_{r} \Lambda^{k}(T)$. Furthermore,

$$
\operatorname{dim} \mathcal{P}_{r}^{-} \Lambda^{k}(T)=\left(\begin{array}{c}
r+k-1 \\
k
\end{array}\right)\left(\begin{array}{l}
n+r \\
n-k
\end{array}\right)
$$

We refer to 4 for more details.

The spaces $\mathcal{P}_{r} \Lambda^{k}(T)$ and $P_{r}^{-} \Lambda^{k}(T)$ are intimately connected through their degrees of freedom. In fact, if $\omega \in \mathcal{P}_{r} \Lambda^{k}(T)$, then $\omega$ is uniquely determined by the functionals

$$
\int_{f} \operatorname{Tr}_{f} \omega \wedge \eta, \quad \eta \in \mathcal{P}_{r+k-\operatorname{dim} f}^{-} \Lambda^{\operatorname{dim} f-k}(f), f \in \Delta(T) .
$$

Here $\operatorname{Tr}_{f} \omega$ is the trace of $\omega$ on the subsimplex $f$, and we have adopted the convention that $\mathcal{P}_{r} \Lambda^{k}(f)$ and $\mathcal{P}_{r+1}^{-} \Lambda^{k}(f)$ are taken to be the empty set if $r$ is negative. Furthermore, $\int_{f} \operatorname{Tr}_{f} \omega \wedge \eta=\omega_{x}$ if $\operatorname{dim} f=0$ and $f$ is equal to a vertex $x$. It can be seen that the number of linearly independent functionals, or degrees of freedom, is exactly equal to the dimension of the space $\mathcal{P}_{r} \Lambda^{k}(T)$; see [4, Section 3]. Similarly, for $\omega \in \mathcal{P}_{r}^{-} \Lambda^{k}(T)$ a set of linearly independent degrees of freedom is given as

$$
\int_{f} \operatorname{Tr}_{f} \omega \wedge \eta, \quad \eta \in \mathcal{P}_{r+k-\operatorname{dim} f-1} \Lambda^{\operatorname{dim} f-k}(f), f \in \Delta(T) .
$$

An important property of the degrees of freedom (3.3) and (3.4) is that for any $f \in \Delta(T)$, with $\operatorname{dim} f \geq k, \operatorname{Tr}_{f} \omega$ is determined by the degrees of freedom associated to $f$ and its subsimplexes.

If $\mathcal{T}_{h}$ is a triangulation of $\Omega$, we let $\Delta_{j}\left(\mathcal{T}_{h}\right)$ denote the set of subsimplexes of dimension $j$ of all $T \in \mathcal{T}_{h}$, and $\Delta\left(\mathcal{T}_{h}\right)$ is the set of subsimplexes associated with $\mathcal{T}_{h}$. The space $\mathcal{P}_{r} \Lambda^{k}\left(\mathcal{T}_{h}\right) \subset L^{2} \Lambda^{k}(\Omega)$ is defined to be the set of all $\omega$ such that $\left.\omega\right|_{T} \in$ $\mathcal{P}_{r} \Lambda^{k}(T)$ for all $T \in \mathcal{T}_{h}$, and such that $\operatorname{Tr}_{f} \omega$ is continuous for all $f \in \Delta_{n-1}\left(\mathcal{T}_{h}\right)$. The space $\mathcal{P}_{r}^{-} \Lambda^{k}\left(\mathcal{T}_{h}\right) \subset L^{2} \Lambda^{k}(\Omega)$ is defined similarly by replacing $\mathcal{P}_{r} \Lambda^{k}(T)$ with $\mathcal{P}_{r}^{-} \Lambda^{k}(T)$. The spaces $\mathcal{P}_{r} \Lambda^{k}\left(\mathcal{T}_{h}\right)$ and $\mathcal{P}_{r}^{-} \Lambda^{k}\left(\mathcal{T}_{h}\right)$ are in fact subspaces of $H \Lambda^{k}(\Omega)$; cf. 4, Theorem 5.1]. 
We will frequently use $\Lambda_{h}^{k}$ to denote subspaces of $H \Lambda^{k}(\Omega)$ that are of the form $\mathcal{P}_{r} \Lambda^{k}\left(\mathcal{T}_{h}\right)$ or $\mathcal{P}_{r}^{-} \Lambda^{k}\left(\mathcal{T}_{h}\right)$. Furthermore, $\Lambda_{h}^{k}(T)$ will be the corresponding polynomial space on the simplex $T \in \mathcal{T}_{h}$. The degrees of freedom given by (3.3) or (3.4) define an interpolation operator $I_{h}: C \Lambda^{k}(\Omega) \rightarrow \Lambda_{h}^{k}$ by the requirement that $F\left(I_{h} \omega\right)=$ $F(\omega)$ for all the functionals $F$ associated with all $f \in \Delta\left(\mathcal{T}_{h}\right)$. Alternatively, if $\mathcal{T}_{h}$ consists of a single simplex $T$ we write $I_{T}$ instead of $I_{h}$. We will refer to the operators $I_{h}$ as the canonical interpolation operators derived from the degrees of freedom. A key property of these interpolation operators is that they commute with the exterior derivative, i.e.

$$
I_{h} \circ d=d \circ I_{h},
$$

if the spaces $\Lambda_{h}^{k}$ and $\Lambda_{h}^{k+1}$ are properly chosen. In fact, the relation (3.5) holds for all the four possible choices where $\Lambda_{h}^{k}=\mathcal{P}_{r} \Lambda^{k}\left(\mathcal{T}_{h}\right)$ or $\Lambda_{h}^{k}=\mathcal{P}_{r}^{-} \Lambda^{k}\left(\mathcal{T}_{h}\right)$, and $\Lambda_{h}^{k+1}=\mathcal{P}_{r}^{-} \Lambda^{k+1}\left(\mathcal{T}_{h}\right)$ or $\Lambda_{h}^{k+1}=\mathcal{P}_{r-1} \Lambda^{k+1}\left(\mathcal{T}_{h}\right)$; cf. [4, Theorem 5.2]. Hence, by combining such choices of spaces, we obtain a discrete de Rham complex and a commuting diagram of the form

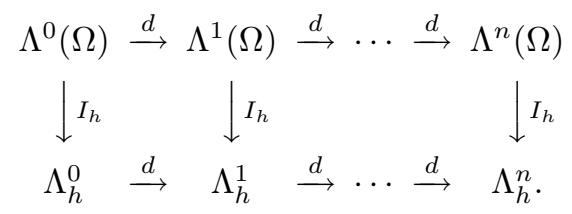

However, the diagram above does not have all the desired properties. Since the Sobolev spaces $H \Lambda^{k}(\Omega)$ frequently occur as the natural solution space for weak formulations of partial differential equations, a more useful diagram is of the form

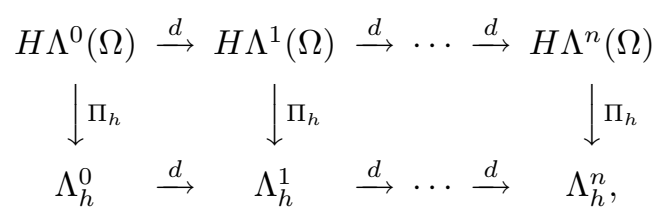

where the operators $\Pi_{h}$ are bounded projections from $H \Lambda^{k}(\Omega)$ onto $\Lambda_{h}^{k}$. The canonical interpolation operators $I_{h}$ do not have this property, since the functions in $H \Lambda^{k}(\Omega)$ do not have well-defined traces on all elements of $\Delta_{j}\left(\mathcal{T}_{h}\right)$ for $j \geq k$. Therefore, there is a need for a construction of such operators. As we have already stated above, such a construction is done in [4] for the case of quasi-uniform triangulations. The operators $\Pi_{h}$ defined in Section 5 below generalize this construction to the non-quasi-uniform case. Furthermore, the case of essential boundary conditions is treated in Section 6 .

Remark. Modified interpolation operators that are both bounded on the spaces $H \Lambda^{k}(\Omega)$ and commute with the exterior derivative were first constructed in [14] in the three-dimensional case. The approach taken there was to average the canonical interpolation operators $I_{h}$ with respect to perturbations of the triangulation $\mathcal{T}_{h}$. An alternative approach, using a standard smoothing operator constructed by a convolution, was then proposed in [6]. A key ingredient is to introduce a two-parameter family of smoothing operators. The construction was based on a combination of smoothing and cut-off operators on the reference macroelements, and allows for non-quasi-uniform meshes, but does not provide an operator that commutes exactly with the exterior derivative. On the other hand, the construction of [4] was 
based on a corresponding regularization by convolution in the physical domain. By combining this approach with an observation made by Schöberl [13, an operator that commutes with the exterior derivative, but requires a quasi-uniform mesh, is obtained. The construction given below leads to exact commutativity and allows non-quasi-uniform meshes.

It will be convenient to introduce a Lipschitz continuous function $g_{h}: \Omega \rightarrow \mathbb{R}^{+}$ to represent the variation of $h_{T}=\operatorname{diam} T$ for $T \in \mathcal{T}_{h}$. In fact, the function $g_{h}$ will be piecewise linear with respect to the mesh $\mathcal{T}_{h}$ and is determined by setting

$$
g_{h}(x)=\frac{1}{\left|\mathcal{T}_{h}(x)\right|} \sum_{T \in \mathcal{T}_{h}(x)} h_{T}, \quad x \in \Delta_{0}\left(\mathcal{T}_{h}\right)
$$

Here $\mathcal{T}_{h}(x)=\left\{T \mid T \in \mathcal{T}_{h}, x \in T\right\}$, and $\left|\mathcal{T}_{h}(x)\right|$ is the number of elements in $\mathcal{T}_{h}(x)$. Clearly, $g_{h}(x) \leq h$, and it is a consequence of the shape regularity (3.1) that there exist positive constants $c_{0}, c_{1}$, independent of $h$, such that

$$
c_{0} g_{h}(x) \leq h_{T} \leq c_{1} g_{h}(x), \quad x \in T \in \mathcal{T}_{h} .
$$

Furthermore, there is a positive constant $L_{\text {mesh }}$, depending on $C_{\text {mesh }}$, such that

$$
\left|g_{h}(x)-g_{h}(y)\right| \leq L_{\text {mesh }}|x-y|, \quad x, y \in \Omega ;
$$

i.e., the functions $\left\{g_{h}\right\}$ are uniformly Lipschitz continuous. To see this, just observe that since $g_{h}$ is piecewise linear and the mesh is shape regular, (3.8) will follow if the bound can be established when $x, y \in \Delta_{0}\left(\mathcal{T}_{h}\right)$ and connected by an edge in $\Delta_{1}\left(\mathcal{T}_{h}\right)$. However, in this case the bound (3.8) follows by (3.2), (3.7) and the triangle inequality.

Remark. We note that the function $g_{h}(x)$, introduced above, in general will depend on $x$ even if the family of triangulations $\left\{\mathcal{T}_{h}\right\}$ is quasi-uniform. However, in this case there is a constant $C_{\text {mesh }}^{\prime}>0$, independent of $x$ and $\mathcal{T}_{h}$, such that $h \geq g_{h}(x) \geq$ $C_{\text {mesh }}^{\prime} h$, and the construction below could as well be carried out with $g_{h}(x)$ replaced by the constant $h$. In this case the smoothing operator $R_{h}^{\epsilon}$, defined by (4.2) below, will reduce to a standard convolution, and the projections $\Pi_{h}$, which will be defined in Section 5, correspond exactly to the projections introduced in [4].

\section{Space-Dependent Smoothing}

Throughout the next two sections the notation $\Lambda_{h}^{k} \subset H \Lambda^{k}(\Omega)$ is used to denote a finite element space of differential forms which is either of the form $\mathcal{P}_{r} \Lambda^{k}\left(\mathcal{T}_{h}\right)$ or $\mathcal{P}_{r}^{-} \Lambda^{k}\left(\mathcal{T}_{h}\right)$, and $I_{h}$ will denote the associated canonical imterpolation operator. The construction of the smoothed projection operators given in 4 was based on operators of the form

$$
I_{h} \circ R_{h}^{\epsilon} \circ E,
$$

where $R_{h}^{\epsilon}$ is a smoothing operator defined by convolution with a mollifier function, and $E$ is an extension operator. The main difference here, from the discussion given in [4], is that the smoothing operator is space dependent. 


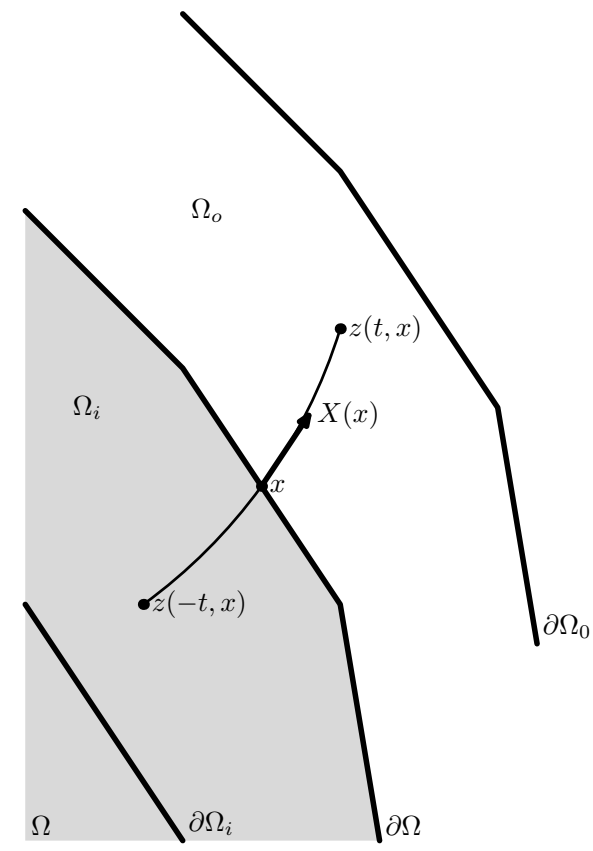

Figure 4.1. Extension by reflection

4.1. The extension operator. Following 14 we will make use of an extension operator $E$, defined by a reflection with respect to the boundary, mapping $H \Lambda^{k}(\Omega)$ into $H \Lambda^{k}(\tilde{\Omega})$. Here $\tilde{\Omega} \supset \bar{\Omega}$, where $\bar{\Omega}$ is the closure of $\Omega$.

Since the domain $\Omega$ is a bounded polygonal Lipschitz domain, we can use standard techniques, involving local coordinates and a partition of unity, to construct a smooth vector field $X$ defined on $\mathbb{R}^{n}$ satisfying $|X(x)| \leq 1$ on $\mathbb{R}^{n}$, and

$$
X(x) \cdot n(x) \geq C_{\Omega}>0, \quad x \in \partial \Omega .
$$

Here $n$ is the outer unit normal vector on the boundary $\partial \Omega$, and $C_{\Omega}$ is a constant which depends on the domain $\Omega$. Consider the curves $z(t)=z(t ; x)$ defined by

$$
\frac{d}{d t} z=X(z), \quad z(0)=x
$$

From the properties of the vector field $X$ it follows that there is a $t_{0}>0$ such that $z(t ; x) \in \mathbb{R}^{n} \backslash \bar{\Omega}$ for $0<t \leq t_{0}$ and $z(t ; x) \in \Omega$ for $-t_{0} \leq t<0$ for any $x \in \partial \Omega$. Define $\Omega_{i} \subset \Omega$ and $\Omega_{o} \subset \mathbb{R}^{n} \backslash \bar{\Omega}$ by

$$
\Omega_{i}=\bigcup_{-t_{0}<t<0} z(t ; \partial \Omega) \quad \text { and } \quad \Omega_{o}=\bigcup_{0<t<t_{0}} z(t ; \partial \Omega) .
$$

The map $\Psi: \Omega_{o} \cup \partial \Omega \rightarrow \Omega_{i} \cup \partial \Omega$ is given by $\Psi(z(t ; x))=z(-t ; x)$ for $0 \leq t<t_{0}$, and $x \in \partial \Omega$ is a Lipschitz continuous bijection, with the additional property that $\Psi(x)=x$ on $\partial \Omega$; cf. Figure 4.1. The desired extension operator $E$ is defined by a reflection with respect to the boundary $\partial \Omega$. We let $\tilde{\Omega}=\bar{\Omega} \cup \Omega_{0}$ and define $E: H \Lambda^{k}(\Omega) \rightarrow H \Lambda^{k}(\tilde{\Omega})$ by

$$
(E \omega)_{x}=\left(\Psi^{*} \omega\right)_{x}, \quad x \in \Omega_{o}
$$


This operator clearly maps $L^{2} \Lambda^{k}(\Omega)$ boundedly into $L^{2} \Lambda^{k}(\tilde{\Omega})$, and since $d \circ \Psi^{*}=$ $\Psi^{*} \circ d$ we have $E \in \mathrm{L}\left(H \Lambda^{k}(\Omega), H \Lambda^{k}(\tilde{\Omega})\right)$. Finally, note that there exists an $\epsilon_{0}>0$ such that $B_{\epsilon}(x) \subset \tilde{\Omega}$ for any $x \in \Omega$ and $0<\epsilon \leq \epsilon_{0}$.

4.2. The smoothing operator. The extension operator introduced above will be used to define smoothing operators, $R_{h}^{\epsilon}$, depending on the mesh $\mathcal{T}_{h}$ and a positive parameter $\epsilon$. Let $B_{1}=B_{1}(0)=\left\{y \in \mathbb{R}^{n}|| y \mid \leq 1\right\}$. For a given $x \in \Omega, y \in B_{1}$, and $0<\epsilon \leq \epsilon_{0}$ define functions $\Phi_{h}^{\epsilon y}: \Omega \rightarrow \tilde{\Omega}$ by

$$
\Phi_{h}^{\epsilon y}(x)=x+\epsilon g_{h}(x) y .
$$

The smoothing operator $R_{h}^{\epsilon}: L^{2} \Lambda^{k}(\Omega) \rightarrow C \Lambda^{k}(\Omega)$ is defined by

$$
\left(R_{h}^{\epsilon} \omega\right)_{x}=\int_{B_{1}} \rho(y)\left(\left(\Phi_{h}^{\epsilon y}\right)^{*} E \omega\right)_{x} d y .
$$

Here $E$ is the extension operator introduced above, and $\rho \in C^{\infty}(\Omega)$ is a fixed mollifier function satisfying

$$
0 \leq \rho(x) \leq 1, \quad \operatorname{supp}(\rho) \subset B_{1}, \quad \int_{B_{1}} \rho(y) d y=1 .
$$

The property that $R_{h}^{\epsilon}$ commutes with the exterior derivative, i.e. $d \circ R_{h}^{\epsilon}=R_{h}^{\epsilon} \circ d$, is an immediate consequence of the corresponding property for the pullbacks $\left(\Phi_{h}^{\epsilon y}\right)^{*}$ and the extension operator $E$. Furthermore, since $\epsilon g_{h}(x)$ is strictly positive and Lipschitz continuous we can conclude that $R_{h}^{\epsilon}\left(L^{2} \Lambda^{k}(\Omega)\right) \subset C \Lambda^{k}(\Omega)$; cf. Lemma 4.1 below. Of course, if the mesh function $g_{h}(x)$ is independent of $x$, and taken equal to $h$, then the operator $R_{h}^{\epsilon}$ reduces to the standard smoothing operator used in [4, defined by convolution with $\rho_{\epsilon h}(x)=\rho(x / \epsilon h) /(\epsilon h)^{n}$.

4.3. Scaling. If $T \in \mathcal{T}_{h}$ we let $\mathcal{T}_{h}(T):=\left\{T^{\prime} \in \mathcal{T}_{h} \mid T^{\prime} \cap T \neq \emptyset\right\}$ be the macroelement in $\mathcal{T}_{h}$ determined by $T$. Furthermore, if $T \cap \partial \Omega=\emptyset$ we let $T^{\star}$ denote the corresponding domain, i.e.

$$
T^{\star}=\bigcup_{T^{\prime} \in \mathcal{T}_{h}(T)} T^{\prime}
$$

see the left part of Figure 4.2. If $T \cap \partial \Omega \neq \emptyset$ the domain $T^{\star}$ is further extended to also include

$$
\left\{x \in \tilde{\Omega} \backslash \Omega \mid \operatorname{dist}(x, T) \leq h_{T}\right\} ;
$$

see the right part of Figure 4.2, It follows from the shape-regularity assumption (3.1) that we can assume that $\epsilon_{0}$ can be chosen sufficiently small such that $\Phi_{h}^{\epsilon y}(x) \in$ $T^{\star}$ for all $x \in T \in \mathcal{T}_{h}$, all $h$, and $0 \leq \epsilon \leq \epsilon_{0}$.

Let $F(x)=F_{T}(x)=\left(x-x_{0}\right) / h_{T}$ where $x_{0}$ is the first vertex of $T$. Thus $F$ maps $T$ onto a simplex $\hat{T}$ with a vertex at the origin and diameter bounded above and below by positive constants depending only on $C_{\text {mesh }}$. It also maps $T^{\star}$ onto $\hat{T}^{\star}:=F\left(T^{\star}\right)$. Furthermore, we let $\mathcal{T}(\hat{T})$ be the set of $n$-simplexes which defines $\hat{T}^{\star}$, i.e.

$$
\mathcal{T}(\hat{T})=\left\{F\left(T^{\prime}\right) \mid T^{\prime} \in \mathcal{T}_{h}(T)\right\} .
$$

The operator $\hat{R}_{h}^{\epsilon}=F^{*-1} R_{h}^{\epsilon} F^{*}: L^{2} \Lambda^{k}\left(\hat{T}^{\star}\right) \rightarrow L^{2} \Lambda^{k}(\hat{T})$ can be identified with a corresponding smoothing operator in the space of scaled variables. In fact, a crucial 

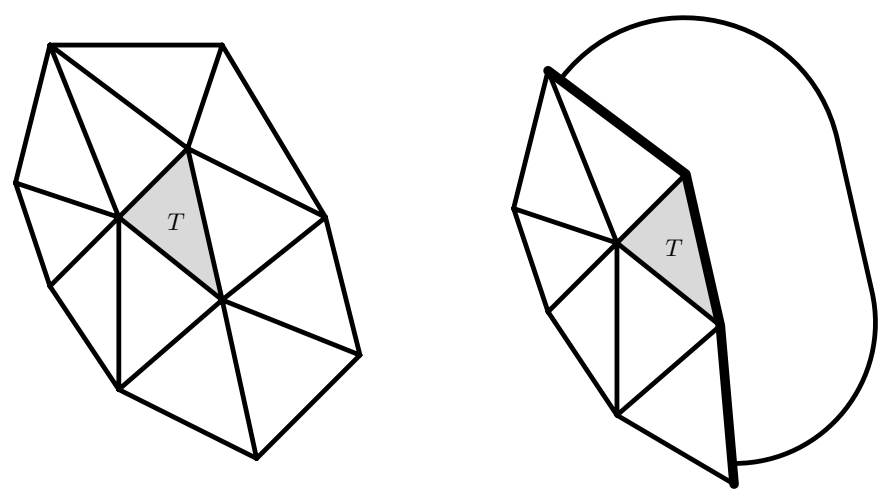

Figure 4.2. The macroelement $\mathcal{T}_{h}(T)$ and the domain $T^{\star}$ associated with the shaded simplex $T$. To the left, $T$ is an interior simplex, while $T$ intersects the boundary, and hence $T^{\star}$ extends outside $\Omega$, to the right.

property of this operator is that it can be properly bounded independently of $h$. We find that

$$
\left(\hat{R}_{h}^{\epsilon} \omega\right)_{x}=\left(F^{*-1} R_{h}^{\epsilon} F^{*} \omega\right)_{x}=\int_{B_{1}} \rho(y)\left(\left(\hat{\Phi}_{h}^{\epsilon y}\right)^{*} \hat{E} \omega\right)_{x} d y
$$

for $\omega \in L^{2} \Lambda^{k}(\hat{T})$. Here, $\hat{E}=F^{*-1} E F^{*}$ and the map $\hat{\Phi}_{h}^{\epsilon y}: \hat{T} \rightarrow \hat{T}^{\star}$ is given by

$$
\hat{\Phi}_{h}^{\epsilon y}(x)=x+\epsilon \hat{g}_{h}(x) y,
$$

where the scaled mesh functions $\hat{g}_{h}$ are given by $\hat{g}_{h}(x)=h_{T}^{-1} g_{h}\left(F^{-1} x\right)$. Observe that (3.7) implies that the functions $\hat{g}_{h}$ are bounded from above and below uniformly with respect to $T \in \mathcal{T}_{h}$ and $h$. Furthermore, by (3.8) the functions $\hat{g}_{h}$ are Lipschitz continuous, with Lipschitz constant $L_{\text {mesh }}$. As a consequence, the matrices $D \hat{\Phi}_{h}^{\epsilon y}$, for $0<\epsilon \leq \epsilon_{0}$ and $y \in B_{1}$, satisfy

$$
\left|D \hat{\Phi}_{h}^{\epsilon y}-I\right| \leq \epsilon L_{\text {mesh }}
$$

on $\hat{T}$, where $|\cdot|$ is the matrix operator norm.

We obtain the following bound, which is uniform with respect to $T \in \mathcal{T}_{h}$ and $h$, on the scaled smoothing operator $\hat{R}_{h}^{\epsilon}$.

Lemma 4.1. For each $\epsilon \in\left(0, \epsilon_{0}\right]$ there is a constant $c(\epsilon)$, independent of $T \in \mathcal{T}_{h}$ and $h$, such that

$$
\left\|\hat{R}_{h}^{\epsilon}\right\|_{E\left(L^{2} \Lambda^{k}\left(\hat{T}^{\star}\right), C \Lambda^{k}(\hat{T})\right)} \leq c(\epsilon) .
$$

Proof. Throughout this proof $\epsilon \in\left(0, \epsilon_{0}\right]$ is fixed. As $T \in \mathcal{T}_{h}$ and $h$ vary, the configuration of simplices in $\hat{T}^{\star}$ varies over a compact set, and hence it is sufficient to show the desired estimate for any single simplex $T$ with a fixed macroelement neighbourhood $T^{\star}$; cf. the proof of Theorem 5.3 of [4]. 
Let $x \in \hat{T}$ be fixed. For $\omega \in L^{2} \Lambda^{k}\left(\hat{T}^{\star}\right)$, and fixed unit vectors $v_{1}, v_{2}, \ldots, v_{k} \in \mathbb{R}^{n}$, we obtain from (4.3) and (4.4) that

$$
\begin{aligned}
\left|\left(\hat{R}_{h}^{\epsilon} \omega\right)_{x}\left(v_{1}, \ldots, v_{k}\right)\right|^{2} & =\left|\int_{B_{1}} \rho(y)\left(\left(\hat{\Phi}_{h}^{\epsilon y}\right)^{*} \hat{E} \omega\right)_{x}\left(v_{1}, \ldots, v_{k}\right) d y\right|^{2} \\
& \leq \int_{B_{1}}\left|\left(\left(\hat{\Phi}_{h}^{\epsilon y}\right)^{*} \hat{E} \omega\right)_{x}\left(v_{1}, \ldots, v_{k}\right)\right|^{2} d y \\
& =(\delta)^{-n} \int_{B_{\delta}(x)}\left|\hat{E} \omega_{z}\left(\left(D \hat{\Phi}_{h}^{\epsilon y}\right) v_{1}, \ldots,\left(D \hat{\Phi}_{h}^{\epsilon y}\right) v_{k}\right)\right|^{2} d z \\
& \leq c\|\omega\|_{L^{2} \Lambda^{k}\left(\hat{T}^{\star}\right)}^{2}
\end{aligned}
$$

where $z=\hat{\Phi}_{h}^{\epsilon y}(x), \delta=\epsilon \hat{g}_{h}(x)$ and $B_{\delta}(x)$ is the ball with centre at $x$ and radius $\delta$. This shows the proper uniform bound on $\hat{R}_{h}^{\epsilon}$ in $\mathrm{L}\left(L^{2} \Lambda^{k}\left(\hat{T}^{\star}\right), C \Lambda^{k}(\hat{T})\right)$.

Remark. Note that Lemma 4.1 will still be true if the extension operator $E$ is taken to be extension by zero outside $\Omega$.

In addition to the smoothing operator $\hat{R}_{h}^{\epsilon}$ we will need a smooth translation map $\hat{\Gamma}^{\epsilon}: \hat{T}^{\dagger} \rightarrow \hat{T}^{\star}$, where the domain $\hat{T}^{\dagger}$ is such that

$$
\hat{T} \subset \hat{T}^{\dagger} \subset \hat{T}^{\star} .
$$

More precisely, we will assume that

$$
\left|\hat{\Gamma}^{\epsilon}(x)-x\right|,\left|D \hat{\Gamma}^{\epsilon}(x)-I\right| \leq c \epsilon,
$$

where the constant $c$ is independent of $T \in \mathcal{T}_{h}, h$ and $\epsilon$. Hence, it is consistent to assume in addition that

$$
B_{\epsilon \hat{g}_{h}(x)}(x) \subset \hat{T}^{\star}, x \in \hat{T}^{\dagger}, \quad \text { and } \hat{T} \subset \hat{\Gamma}^{\epsilon}\left(\hat{T}^{\dagger}\right)
$$

for $\epsilon$ sufficiently small.

Lemma 4.2. Let $\omega \in H \Lambda^{k}\left(\hat{T}^{\star}\right)$ with $\left.\omega\right|_{T^{\prime}} \in W_{\infty}^{1} \Lambda^{k}\left(T^{\prime}\right)$ for $T^{\prime} \in \mathcal{T}(\hat{T})$. There is a constant $c$, independent of $T \in \mathcal{T}_{h}$ and $h$ and $\epsilon$, such that

$$
\left\|I_{\hat{T}}\left(I-\hat{\Gamma}^{\epsilon *} \hat{R}_{h}^{\epsilon}\right) \omega\right\|_{L^{2} \Lambda^{k}(\hat{T})} \leq c \epsilon \sum_{T^{\prime} \in \mathcal{T}(\hat{T})}\|\omega\|_{W_{\infty}^{1}\left(T^{\prime}\right)}
$$

for $\epsilon$ sufficiently small. Here the map $\hat{\Gamma}^{\epsilon}$ is assumed to satisfy (4.5) and (4.6).

Proof. Recall that the space $\Lambda_{h}^{k}(\hat{T})$ is either of the form $\mathcal{P}_{r} \Lambda^{k}(\hat{T})$ or $\mathcal{P}_{r}^{-} \Lambda^{k}(\hat{T})$. As a consequence, the canonical interpolation operator $I_{\hat{T}}$ is determined by moments with respect to the subsimplexes $f$ of $\hat{T}$ with $\operatorname{dim} f \geq k$. More precisely, it is enough to show that for a given $f \in \Delta(\hat{T})$, with $\operatorname{dim} f \geq k$, and $\eta \in \Lambda^{\operatorname{dim} f-k}(f)$ we have

$$
\left|\int_{f}\left(I-\hat{\Gamma}^{\epsilon *} \hat{R}_{h}^{\epsilon}\right) \omega \wedge \eta\right| \leq c \epsilon \sum_{T^{\prime} \in \mathcal{T}(\hat{T})}\|\omega\|_{W_{\infty}^{1}\left(T^{\prime}\right)}
$$

for all $\omega \in H \Lambda^{k}\left(\hat{T}^{\star}\right)$ such that $\left.\omega\right|_{T^{\prime}} \in W_{\infty}^{1} \Lambda^{k}\left(T^{\prime}\right)$ for $T^{\prime} \in \mathcal{T}(\hat{T})$. Here the constant $c$ is independent of $\epsilon$ and $\omega$, but it is allowed to depend on the test function $\eta$.

Let us first note that if $\operatorname{dim} f=0$, so that $f$ is equal to a vertex $x$, then $k=0$ and $\omega$ is continuous at $x$. Furthermore, the estimate (4.7) follows from the bound

$$
\left|\omega_{x}-\omega_{z}\right| \leq|x-z| \sum_{T^{\prime} \in \mathcal{T}(\hat{T})}\|\omega\|_{W_{\infty}^{1}\left(T^{\prime}\right)} .
$$


To show the bound (4.7) when $\operatorname{dim} f>0$ we will decompose the face $f$ into $f_{\epsilon}$ and $f \backslash f_{\epsilon}$, where

$$
f_{\epsilon}=\{x \in f \mid \operatorname{dist}(x, \partial f) \geq C \epsilon\} .
$$

Here the constant $C>0$ is chosen such that for any point $x \in f_{\epsilon}$, the set $\hat{\Gamma}^{\epsilon}\left(B_{\epsilon}(x)\right)$ will only intersect the elements of $\mathcal{T}(\hat{T})$ that have $f$ as a subsimplex. A consequence of this construction is that if $x \in f_{\epsilon}, y \in B_{1}, z=\hat{\Gamma}^{\epsilon} \circ \hat{\Phi}_{h}^{\epsilon y}(x)$ and $v_{1}, v_{2}, \ldots, v_{k}$ are unit tangent vectors to $f$, then it follows from (4.4) and (4.5) that

$$
\begin{aligned}
\mid \omega_{x}\left(v_{1}, \ldots, v_{k}\right) & -\omega_{z}\left(D\left(\hat{\Gamma}^{\epsilon} \circ \hat{\Phi}_{h}^{\epsilon y}\right) v_{1}, \ldots, D\left(\hat{\Gamma}^{\epsilon} \circ \hat{\Phi}_{h}^{\epsilon y}\right) v_{k}\right) \mid \\
& \leq\left(|x-z|+\left|D\left(\hat{\Gamma}^{\epsilon} \circ \hat{\Phi}_{h}^{\epsilon y}\right)-I\right|\right) \sum_{T^{\prime} \in \mathcal{T}(\hat{T})}\|\omega\|_{W_{\infty}^{1}\left(T^{\prime}\right)} \\
& \leq c \epsilon \sum_{T^{\prime} \in \mathcal{T}(\hat{T})}\|\omega\|_{W_{\infty}^{1}\left(T^{\prime}\right)} .
\end{aligned}
$$

However, this implies that

$$
\left|\int_{f_{\epsilon}}\left(I-\hat{\Gamma}^{\epsilon *} \hat{R}_{h}^{\epsilon}\right) \omega \wedge \eta\right| \leq c \epsilon \sum_{T^{\prime} \in \mathcal{T}(\hat{T})}\|\omega\|_{W^{1, \infty}\left(T^{\prime}\right)},
$$

where the constant $c$ is independent of $\epsilon$ and $\omega$. Finally, it is straightforward to see that

$$
\left|\int_{f \backslash f_{\epsilon}}\left(I-\hat{\Gamma}^{\epsilon *} \hat{R}_{h}^{\epsilon}\right) \omega \wedge \eta\right| \leq\left|\int_{f \backslash f_{\epsilon}} \omega \wedge \eta\right|+\left|\int_{f \backslash f_{\epsilon}} \hat{\Gamma}^{\epsilon *} \hat{R}_{h}^{\epsilon} \omega \wedge \eta\right| \leq c \epsilon\|\omega\|_{L^{\infty} \Lambda^{k}\left(\hat{T}^{\star}\right)} .
$$

Hence we have verified the bound (4.7) when $\operatorname{dim} f>0$. This completes the proof.

\section{The SMOOTHED PROJECTION}

The canonical interpolation operator $I_{h}$ and the smoothing operators $R_{h}^{\epsilon}$ introduced above will be used to define a projection operator $\Pi_{h}$ onto the finite element space $\Lambda_{h}^{k}$ which is uniformly bounded with respect to $h$ in both $L^{2} \Lambda^{k}(\Omega)$ and $H \Lambda^{k}(\Omega)$. The following results, which correspond to Lemma 5.4 of [4, are crucial for this construction.

Proposition 5.1. For each $\epsilon \in\left(0, \epsilon_{0}\right]$ there exists a constant $c(\epsilon)$ such that

$$
\left\|I_{h} R_{h}^{\epsilon}\right\|_{E\left(L^{2} \Lambda^{k}(\Omega), L^{2} \Lambda^{k}(\Omega)\right)} \leq c(\epsilon)
$$

for all $h$.

Proof. As above we let $T^{\star}=\bigcup\left\{T^{\prime} \mid T^{\prime} \in \mathcal{T}_{h}(T)\right\}$ denote the macroelement associated with $T \in \mathcal{T}_{h}$. We shall write $\Lambda_{h}^{k}(T)$ and $\Lambda_{h}^{k}\left(T^{\star}\right)$ for the space of restrictions of elements of $\Lambda_{h}^{k}$ to $T$ or $T^{\star}$. The shape regularity property implies bounded overlap of the $T^{\star}$, so

$$
\sum_{T \in \mathcal{T}_{h}}\|\omega\|_{L^{2} \Lambda^{k}\left(T^{\star}\right)}^{2} \leq c\|\omega\|_{L^{2} \Lambda^{k}(\Omega)}^{2} .
$$

Therefore, to complete the proof it suffices to show that

$$
\left\|I_{T} R_{h}^{\epsilon}\right\|_{\mathrm{E}\left(L^{2} \Lambda^{k}\left(T^{\star}\right), L^{2} \Lambda^{k}(T)\right)} \leq c(\epsilon)
$$

with $c(\epsilon)$ uniform over $T \in \mathcal{T}_{h}$ and over $h$.

Let $F(x)=F_{T}(x)=\left(x-x_{0}\right) / h_{T}$, where $x_{0}$ is the first vertex of $T$, be a scaling of $T$ as described in Section 4 above. Thus $F$ maps $T$ onto a simplex $\hat{T}$ with a vertex 
at the origin and it maps $T^{\star}$ onto $\hat{T}^{\star}:=F_{T}\left(T^{\star}\right)$. Then $F^{*-1} I_{T} F^{*}: \Lambda^{k}(\hat{T}) \rightarrow \Lambda_{h}^{k}(\hat{T})$ is just the canonical interpolation operator $I_{\hat{T}}$ onto the polynomial space $\Lambda_{h}^{k}(\hat{T})$. In particular, $I_{\hat{T}} \in \mathrm{E}\left(C \Lambda^{k}(\hat{T}), L^{2} \Lambda^{k}(\hat{T})\right)$, and, as in the proof of Lemma 4.1 above, the compactness of the simplices $\hat{T}$ implies that the corresponding operator norm is bounded independently of $T \in \mathcal{T}_{h}$ and $h$. Furthermore,

$$
\begin{aligned}
\left\|I_{T} R_{h}^{\epsilon}\right\|_{\mathrm{E}\left(L^{2} \Lambda^{k}\left(T^{\star}\right), L^{2} \Lambda^{k}(T)\right)} & =\left\|F^{*-1} I_{T} R_{h}^{\epsilon} F^{*}\right\|_{\mathrm{E}\left(L^{2} \Lambda^{k}\left(\hat{T}^{\star}\right), L^{2} \Lambda^{k}(\hat{T})\right)} \\
& =\left\|I_{\hat{T}} \hat{R}_{h}^{\epsilon}\right\|_{\mathrm{E}\left(L^{2} \Lambda^{k}\left(\hat{T}^{\star}\right), L^{2} \Lambda^{k}(\hat{T})\right)},
\end{aligned}
$$

where we recall that $\hat{R}_{h}^{\epsilon}=F^{*-1} R_{h}^{\epsilon} F^{*}$. Hence, since $I_{\hat{T}}$ is uniformly bounded in $\mathrm{E}\left(C \Lambda^{k}(\hat{T}), L^{2} \Lambda^{k}(\hat{T})\right)$, the desired bound (5.1) follows from Lemma 4.1,

Due to Proposition 5.1 the operators $I_{h} R_{h}^{\epsilon}$ map $L^{2} \Lambda^{k}(\Omega)$ onto $\Lambda_{h}^{k}$ and they are uniformly bounded with respect to $h$ for a fixed $\epsilon>0$. However, these operators are not projections since they are not invariant on $\Lambda_{h}^{k}$. The next result, which generalizes Lemma 5.5 of [4, will be essential to modify the operators $I_{h} R_{h}^{\epsilon}$ into projections. This construction of a projection is inspired by [13, Theorem 7].

Proposition 5.2. There exists a constant $c$, independent of $\epsilon$ and $h$ such that

$$
\left\|I-\left.I_{h} R_{h}^{\epsilon}\right|_{\Lambda_{h}^{k}}\right\|_{E\left(L^{2} \Lambda_{h}^{k}, L^{2} \Lambda_{h}^{k}\right)} \leq c \epsilon
$$

for $0<\epsilon \leq \epsilon_{0}$. Here $\|\cdot\|_{E\left(L^{2} \Lambda_{h}^{k}, L^{2} \Lambda_{h}^{k}\right)}$ denotes the $L^{2}$ operator norm of an operator $\Lambda_{h}^{k} \rightarrow \Lambda_{h}^{k}$.

Proof. As in the proof of Proposition 5.1 above it is enough to show the estimate locally; i.e., the desired result will follow from a local bound of the form

$$
\left\|I-I_{T} R_{h}^{\epsilon}\right\|_{\mathrm{E}\left(L^{2} \Lambda_{h}^{k}\left(T^{\star}\right), L^{2} \Lambda_{h}^{k}(T)\right)} \leq c \epsilon,
$$

where the constant $c$ is uniform with respect to $T \in \mathcal{T}_{h}$ and $h$. Define the scaling $F(x)=F_{T}(x)=\left(x-x_{0}\right) / h_{T}$ as above. Then

$$
\left\|I-I_{T} R_{h}^{\epsilon}\right\|_{\mathrm{E}\left(L^{2} \Lambda_{h}^{k}\left(T^{\star}\right), L^{2} \Lambda_{h}^{k}(T)\right)}=\left\|I-I_{\hat{T}} \hat{R}_{h}^{\epsilon}\right\|_{\mathrm{E}\left(L^{2} \Lambda_{h}^{k}\left(\hat{T}^{\star}\right), L^{2} \Lambda_{h}^{k}(\hat{T})\right)} .
$$

We therefore conclude that the desired estimate will follow if we can show that

$$
\left\|I-I_{\hat{T}} \hat{R}_{h}^{\epsilon}\right\|_{\mathrm{E}\left(L^{2} \Lambda_{h}^{k}\left(\hat{T}^{\star}\right), L^{2} \Lambda_{h}^{k}(\hat{T})\right)} \leq c \epsilon .
$$

However, if $\omega \in \Lambda_{h}^{k}\left(\hat{T}^{\star}\right)$, then $I_{\hat{T}} \omega=\left.\omega\right|_{\hat{T}}$ and by the compactness of the macroelements $\mathcal{T}_{h}(\hat{T})$ we have

$$
\sum_{T^{\prime} \in \mathcal{T}(\hat{T})}\|\omega\|_{W_{\infty}^{1}\left(T^{\prime}\right)} \leq c\|\omega\|_{L^{2} \Lambda_{h}^{k}\left(\hat{T}^{\star}\right)} .
$$

Therefore, the bound (5.2) follows directly from Lemma 4.2 with $\hat{\Gamma}^{\epsilon}$ taken to be the identity map.

It follows from Proposition 5.2 that there is an $\epsilon_{1}, 0<\epsilon_{1} \leq \epsilon_{0}$ such that the operator $\left.I_{h} R_{h}^{\epsilon}\right|_{\Lambda_{h}^{k}}$ is invertible for $0<\epsilon \leq \epsilon_{1}$, and such that its inverse, $J_{h}^{\epsilon}$, satisfies

$$
\left\|J_{h}^{\epsilon}\right\|_{\mathrm{E}\left(L^{2} \Lambda_{h}^{k}, L^{2} \Lambda_{h}^{k}\right)} \leq 2 .
$$

Furthermore, the operator $J_{h}^{\epsilon}$ commutes with the exterior derivative $d$. 
For the rest of the discussion of this section we fix $\epsilon \in\left(0, \epsilon_{1}\right]$. The smoothed interpolation operator $\Pi_{h}: \Lambda_{h}^{k} \rightarrow \Lambda_{h}^{k}$ is now defined by

$$
\Pi_{h}=J_{h}^{\epsilon} I_{h} R_{h}^{\epsilon} .
$$

By construction, this operator is a projection $\left(\Pi_{h}^{2}=\Pi_{h}\right)$, it commutes with the exterior derivative $d$, and it is uniformly bounded in $\mathrm{E}\left(L^{2} \Lambda^{k}(\Omega), L^{2} \Lambda^{k}(\Omega)\right)$. Furthermore, since it commutes with $d$ it is also uniformly bounded in $\mathrm{E}\left(H \Lambda^{k}(\Omega), H \Lambda^{k}(\Omega)\right)$. As in Theorem 5.6 of [4] we also obtain the convergence result below.

Corollary 5.3. The projections $\Pi_{h}$ are uniformly bounded operators in $E\left(L^{2} \Lambda^{k}(\Omega), L^{2} \Lambda^{k}(\Omega)\right)$ and $E\left(H \Lambda^{k}(\Omega), H \Lambda^{k}(\Omega)\right)$ and commute with the exterior derivative d. Moreover, for all $\omega \in L^{2} \Lambda^{k}(\Omega), \Pi_{h} \omega \rightarrow \omega$ in $L^{2}$ as $h \rightarrow 0$.

Remark. By using the bounds on the best approximation given in Theorem 5.3 of 4 we can also obtain rate-of-convergence results for the projection error. If $\mathcal{P}_{r} \Lambda^{k}\left(\mathcal{T}_{h}\right) \subset \Lambda_{h}^{k}$, then

$$
\left\|\omega-\Pi_{h} \omega\right\|_{L^{2} \Lambda^{k}(\Omega)} \leq c h^{s}\|\omega\|_{H^{s} \Lambda^{k}(\Omega)}, \quad \omega \in H^{s} \Lambda^{k}(\Omega), 0 \leq s \leq r+1 ;
$$

cf. Theorem 5.6 of 4 .

\section{ESSENTIAL BOUNDARY CONDITIONS}

The purpose of this section is to construct projection operators, corresponding to the operators $\Pi_{h}$ constructed in Section 5 above, but for the case of essential boundary conditions. As above, the finite element space $\Lambda_{h}^{k}$ is either of the form $\mathcal{P}_{r} \Lambda^{k}\left(\mathcal{T}_{h}\right)$ or $\mathcal{P}_{r}^{-} \Lambda^{k}\left(\mathcal{T}_{h}\right)$, but in this case we also assume that $\Lambda_{h}^{k}$ is a subspace of $\stackrel{\circ}{H} \Lambda^{k}(\Omega)$. The smoothing operators $R_{h}^{\epsilon}$, introduced in Section 4 , will be a key component of the construction. However, in the present section we assume that the extension operator $E$, appearing in the definition (4.2), is taken to be the extension by zero outside $\Omega$.

The vector field $X=X(x)$, introduced in Section 4 above, will be used to define a family of domains $\left\{\Omega_{h}^{\epsilon}\right\}$ such that $\Omega \subset \Omega_{h}^{\epsilon}$, and such that $\Omega_{h}^{\epsilon}$ converges to $\Omega$ as $\epsilon$ or $h$ tends to zero. It follows from the properties of the vector field $X$ that there are fixed positive constants $\delta$ and $t_{1}$ such that the balls $B_{t}(x+\delta t X(x))$ are entirely in $\mathbb{R}^{n} \backslash \Omega$ for all $x \in \partial \Omega$ and $0<t \leq t_{1}$; cf. Figure 6.1 We let

$$
\Omega_{h}^{\epsilon}=\left\{x+\delta t X(x) \mid x \in \Omega, t \leq \epsilon g_{h}(x)\right\},
$$

and let $\Gamma_{h}^{\epsilon}: \Omega \rightarrow \Omega_{h}^{\epsilon}$ be the corresponding map given by

$$
\Gamma_{h}^{\epsilon}(x)=x+\delta \epsilon g_{h}(x) X(x) .
$$

It follows that $R_{h}^{\epsilon}$ maps the space $\stackrel{\circ}{H} \Lambda^{k}(\Omega)$ boundedly into the space $\stackrel{\circ}{C} \Lambda^{k}\left(\Omega_{h}^{\epsilon}\right) \equiv$ $\stackrel{\circ}{H} \Lambda^{k}\left(\Omega_{h}^{\epsilon}\right) \cap C \Lambda^{k}\left(\Omega_{h}^{\epsilon}\right)$. Furthermore, $\Gamma_{h}^{\epsilon *}$ maps $\stackrel{\circ}{C} \Lambda^{k}\left(\Omega_{h}^{\epsilon}\right)$ into $\stackrel{\circ}{C} \Lambda^{k}(\Omega)$. Therefore, we can conclude that the composition $\Gamma_{h}^{\epsilon *} \circ R_{h}^{\epsilon}$ is in $\mathrm{E}\left(\stackrel{\circ}{H} \Lambda^{k}(\Omega), \stackrel{C}{C} \Lambda^{k}(\Omega)\right)$, and, as a consequence, the map

$$
I_{h} \circ \Gamma_{h}^{\epsilon *} \circ R_{h}^{\epsilon}
$$

is in $\mathrm{E}\left(\stackrel{\circ}{H} \Lambda^{k}(\Omega), \stackrel{\circ}{H} \Lambda^{k}(\Omega)\right)$. Here $I_{h}$ is the canonical interpolation operator onto $\Lambda_{h}^{k}$.

In order to obtain the proper bounds on the operator $I_{h} \circ \Gamma_{h}^{\epsilon *} \circ R_{h}^{\epsilon}$ we consider the scaling of the map $\Gamma_{h}^{\epsilon}$; i.e., we consider $\hat{\Gamma}_{h}^{\epsilon}=F \circ \Gamma_{h}^{\epsilon} \circ F^{-1}$, where $F(x)=$ $F_{T}(x)=\left(x-x_{0}\right) / h_{T}$ is the scaling map associated with $T \in \mathcal{T}_{h}$. More explicitly,

$$
\hat{\Gamma}_{h}^{\epsilon}(x)=x+\left(\delta \epsilon g_{h}(\check{x}) / h_{T}\right) X(\check{x}),
$$




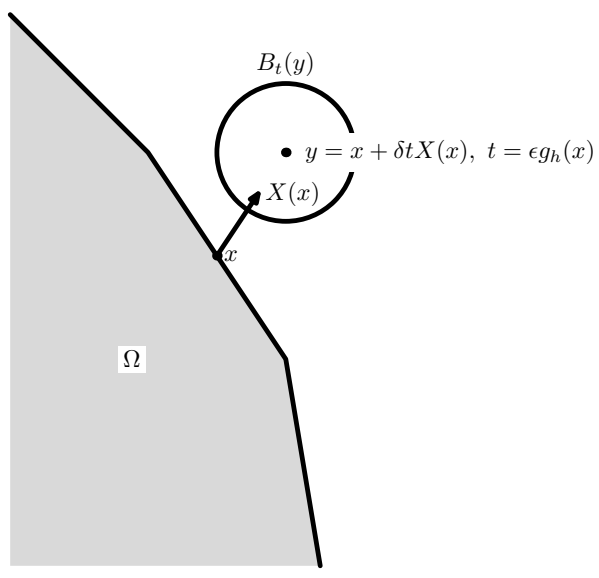

FiguRE 6.1

where $\check{x}=F^{-1}(x)$. Since $g_{h}(x) / h_{T}$ and the Lipschitz constant of $g_{h}$ are bounded independently of $T \in \mathcal{T}_{h}, h$, and $\epsilon$, it follows that the map $\hat{\Gamma}_{h}^{\epsilon}$ satisfies

$$
\left|\hat{\Gamma}_{h}^{\epsilon}(x)-x\right|,\left|D \hat{\Gamma}_{h}^{\epsilon}(x)-I\right| \leq c \epsilon,
$$

where the constant is independent of $T \in \mathcal{T}_{h}, h$, and $\epsilon$. Also observe that we can choose $\epsilon$ sufficiently small such that there is a domain $\hat{T}^{\dagger}, \hat{T} \subset \hat{T}^{\dagger} \subset \hat{T}^{\star}$, with the properties that

$$
B_{\epsilon \hat{g}_{h}(x)}(x) \subset \hat{T}^{\star}, x \in \hat{T}^{\dagger} \quad \text { and } \quad \hat{T} \subset \hat{\Gamma}_{h}^{\epsilon}\left(\hat{T}^{\dagger}\right) .
$$

Therefore, we have seen that the map $\hat{\Gamma}_{h}^{\epsilon}$ satifies the estimates (4.5) and (4.6).

Proposition 6.1. For $\epsilon$ sufficiently small there exists a constant $c(\epsilon)$ such that

$$
\left\|I_{h} \Gamma_{h}^{\epsilon *} R_{h}^{\epsilon}\right\|_{L\left(L^{2} \Lambda^{k}(\Omega), L^{2} \Lambda^{k}(\Omega)\right)} \leq c(\epsilon)
$$

for all $h$.

Proof. The proof follows from a minor variation of the proof of Proposition 5.1 given above. As above we introduce the scaling $F(x)=F_{T}(x)=\left(x-x_{0}\right) / h_{T}$ for each $T \in \mathcal{T}_{h}$, and we observe, in parallel to the proof above, that the desired bound will follow if we can show that $\left\|I_{\hat{T}} \hat{\Gamma}_{h}^{\epsilon *} \hat{R}_{h}^{\epsilon}\right\|_{\mathrm{E}\left(L^{2} \Lambda^{k}\left(\hat{T}^{\star}\right), L^{2} \Lambda^{k}(\hat{T})\right)}$ are bounded uniformly with respect to $T \in \mathcal{T}_{h}$ and $h$. Let the domain $\hat{T}^{\dagger}, \hat{T} \subset \hat{T}^{\dagger} \subset \hat{T}^{\star}$, be as above. As in the proof of Proposition 5.1 we can conclude that

$$
\left\|\hat{R}_{h}^{\epsilon}\right\|_{\mathrm{E}\left(L^{2} \Lambda^{k}\left(T^{\star}\right), C \Lambda^{k}\left(\hat{T}^{\dagger}\right)\right)} \quad \text { and } \quad\left\|I_{\hat{T}}\right\|_{\mathrm{E}\left(C \Lambda^{k}(\hat{T}), L^{2} \Lambda^{k}(\hat{T})\right)}
$$

are bounded uniformly with respect to $T \in \mathcal{T}_{h}$ and $h$. Furthermore, the operator $\hat{\Gamma}_{h}^{\epsilon *}$ is uniformly bounded in $\mathrm{\textrm {L }}\left(C \Lambda^{k}\left(\hat{T}^{\dagger}\right), C \Lambda^{k}(\hat{T})\right)$, since this operator corresponds to a translation along smooth curves; cf. (6.2). This completes the proof.

Proposition 6.2. There exists a constant $c$, independent of $\epsilon, \delta$ and $h$ such that

$$
\left\|I-I_{h} \Gamma_{h}^{\epsilon *} R_{h}^{\epsilon}\right\|_{L\left(L^{2} \Lambda_{h}^{k}, L^{2} \Lambda_{h}^{k}\right)} \leq c \epsilon
$$

for $\epsilon$ sufficiently small. Here $\|\cdot\|_{E\left(L^{2} \Lambda_{h}^{k}, L^{2} \Lambda_{h}^{k}\right)}$ denotes the $L^{2}$ operator norm of an operator $\Lambda_{h}^{k} \rightarrow \Lambda_{h}^{k}$. 
Proof. Since the map $\hat{\Gamma}_{h}^{\epsilon}$ satisfies the estimates (4.5) and (4.6), we can argue, as in the proof of Proposition 5.2 above, and conclude from Lemma 4.2 that

$$
\left\|\left(I-I_{\hat{T}} \hat{\Gamma}_{h}^{\epsilon *} \hat{R}_{h}^{\epsilon}\right) \omega\right\|_{L^{2} \Lambda^{k}(\hat{T})} \leq c \epsilon \sum_{T^{\prime} \in \mathcal{T}(\hat{T})}\|\omega\|_{W_{\infty}^{1}\left(T^{\prime}\right)} \leq c^{\prime} \epsilon\|\omega\|_{L^{2} \Lambda^{k}\left(\hat{T}^{\star}\right)}
$$

for any $\omega \in \Lambda_{h}^{k}$. Hence the desired result follows in complete analogy with the proof of Proposition 5.2

It is a consequence of Proposition 6.2 that the operator $I_{h} \Gamma_{h}^{\epsilon *} R_{h}^{\epsilon}$ restricted to $\Lambda_{h}^{k}$ is invertible for $\epsilon$ sufficiently small. Fixing such an $\epsilon$ we define the smoothed projection as

$$
\Pi_{h}=J_{h}^{\epsilon} I_{h} \Gamma_{h}^{\epsilon} R_{h}^{\epsilon},
$$

where $J_{h}^{\epsilon}=\left(\left.I_{h} \Gamma_{h}^{\epsilon} R_{h}^{\epsilon}\right|_{\Lambda_{h}^{k}}\right)^{-1}$. As in the previous section we obtain the following bound for the projection error.

Corollary 6.3. The projections $\Pi_{h}$ are uniformly bounded operators in $E\left(L^{2} \Lambda^{k}(\Omega), L^{2} \Lambda^{k}(\Omega)\right)$ and $E\left(\stackrel{\circ}{H} \Lambda^{k}(\Omega), \stackrel{\circ}{H} \Lambda^{k}(\Omega)\right)$ and commute with the exterior derivative d. Moreover, for all $\omega \in L^{2} \Lambda^{k}(\Omega), \Pi_{h} \omega \rightarrow \omega$ in $L^{2}$ as $h \rightarrow 0$.

\section{ACKNOWLEDGEMENTS}

The second author has benefited substantially from an ongoing cooperation with Douglas N. Arnold and Richard S. Falk on finite element exterior calculus.

\section{REFERENCES}

[1] D. N. Arnold, Differential complexes and numerical stability, in Proceedings of the International Congress of Mathematicians, Vol. I, Higher Ed. Press, Beijing, 2002, pp. 137-157. MR.1989182(2004h:65115)

[2] D. N. Arnold, R. S. Falk and R. Winther, Differential complexes and stability of finite element methods. I: The de Rham complex, in Compatible Spatial Discretizations, Vol. 142 of The IMA Volumes in Mathematics and its Applications, Springer, Berlin, 2006, pp. 23-46. MR2256572 (2007c:65004)

[3] D. N. Arnold, R. S. Falk and R. Winther, Differential complexes and stability of finite element methods. II: The elasticity complex, in Compatible Spatial Discretizations, Vol. 142 of The IMA Volumes in Mathematics and its Applications, Springer, Berlin, 2006, pp. 47-68. MR2249345

[4] D. N. Arnold, R. S. Falk and R. Winther, Finite element exterior calculus, homological techniques, and applications, Acta Numerica 15 (2006), pp. 1-155. MR2269741

[5] A. Bossavit, Whitney forms: A class of finite elements for three-dimensional computations in electromagnetism, IEE Trans. Mag. 135, Part A (1988), pp. 493-500.

[6] S. H. Christiansen, Stability of Hodge decompositions in finite element spaces of differential forms in arbitrary dimensions, Numer. Math. 107 (2007), pp. 87-106.

[7] J. Dodziuk and V. K. Patodi, Riemannian structures and triangulations of manifolds, Journal of the Indian Math. Soc. 40 (1976), pp. 1-52. MR0488179 (58:7742)

[8] R. Hiptmair, Canonical construction of finite elements, Math. Comp. 68 (1999), pp. 13251346. MR1665954 (2000b:65214)

[9] R. Hiptmair, Higher order Whitney forms, in Geometrical methods in computational electromagnetics (F. Teixeira, ed.), Vol. 32 of PIER, EMW Publishing, Cambridge, MA, pp. $271-299$

[10] R. Hiptmair, Finite elements in computational electromagnetism, Acta Numer. 11 (2002), pp. 237-339. MR.2009375 (2004k:78028)

[11] J.-C. Nédélec, Mixed finite elements in $\mathbf{R}^{3}$, Numer. Math. 35 (1980), pp. 315-341. MR592160 (81k:65125)

[12] J.-C. Nédélec, A new family of mixed finite elements in $\mathbf{R}^{3}$, Numer. Math. 50 (1986), pp. 57-81. MR864305 (88e:65145) 
[13] J. Schöberl, A multilevel decomposition result in $H$ (curl), Proceedings from the 8th European Multigrid, Multilevel, and Multiscale Conference, Scheveningen, The Hague, 2005.

[14] J. Schöberl, A posteriori error estimates for Maxwell equations, RICAM-Report No. 2005-10. To appear in Math. Comp. (2008)

[15] H. Whitney, Geometric integration theory, Princeton University Press, 1957. MR 0087148 $(19: 309 \mathrm{c})$

Centre of Mathematics for Applications and Department of Mathematics, University of Oslo, P.O. Box 1053, Blindern, 0316 Oslo, Norway

E-mail address: snorrec@cma.uio.no

$U R L:$ http://folk.uio.no/ ${ }^{\sim}$ snorrec/

Centre of Mathematics for Applications and Department of Informatics, University of Oslo, P.O. Box 1053, Blindern, 0316 Oslo, Norway

E-mail address: ragnar.winther@cma.uio.no

$U R L:$ http://folk.uio.no/〜rwinther/ 